\title{
A Boundary Value Problem for the Two Dimensional Broadwell Model`
}

\author{
Carlo Cercignani ${ }^{1}$, Reinhard Illner ${ }^{2}$ and Marvin Shinbrot ${ }^{2}$ \\ 1 Politecnico di Milano, I-20133 Milano, Italy \\ 2 University of Victoria, Victoria, Canada V8W 2Y2
}

\begin{abstract}
It is shown that a certain boundary value problem for the steady twodimensional Broadwell model on a rectangle has a solution. The boundary conditions specify the ingoing particle densities on each side of the rectangle.
\end{abstract}

\section{Introduction}

Very little is known about boundary value problems for the Boltzmann equation, even for steady flows. The linearized equation has been much studied $[1,2,4-9$, 13], and there are some results for nonlinear flows near to equilibrium, but that is all we know of, in general. Recently, in an attempt to make further progress on the problem, we began to study boundary value problems for discrete velocity models of the Boltzmann equation. In [11], we showed that, in one dimension, the boundary value problem associated with discrete velocity problems in a slab has solutions quite generally, although we were unable to prove any kind of uniqueness for the solutions we found. In [12], we extended the results of [11] to discrete velocity flows in a half-line. We obtained the result, expected because of the physical analogy, that the solution at infinity is a Maxwellian.

Naturally, one-dimensional steady problems are problems involving ordinary differential equations. In two dimensions, for discrete velocity flows in a domain, virtually nothing is known. In this paper, we present a non-trivial example of the solution of a boundary value problem associated with a natural 4-velocity model in a rectangle. This is the first example we know of such a result.

The model is easily described. We solve the following problem in the rectangle $R=[0, a] \times[0, b]:$

$$
\frac{\partial f^{1}}{\partial x}+f^{1} f^{2}=f^{3} f^{4}, \quad f^{1}(0, y)=\varphi^{1}(y)
$$

\footnotetext{
* Research supported by the Natural Science and Engineering Research Council Canada under Grants A7847 and A8560
} 


$$
\begin{aligned}
-\frac{\partial f^{2}}{\partial x}+f^{1} f^{2} & =f^{3} f^{4}, \\
\frac{\partial f^{3}}{\partial y}+f^{3} f^{4} & =f^{1} f^{2},
\end{aligned}
$$

The underlying time-dependent model associated with (1.1-4) is well known as the (two-dimensional) Broadwell model $[13,15]$. This is one of the simplest models for which the global existence problem for the Cauchy problem is unsolved for large $\operatorname{data}^{1}$ (see [16]). In spite of this, the solution of the boundary value problem (1.1-4) that we present here is, like the results of [11] and [12], completely global, in no way depending on the lengths of the intervals $(0, a)$ and $(0, b)$, or the size of the data $\varphi^{1}, \varphi^{2}, \varphi^{3}$, or $\varphi^{4}$.

A piece of notation before we start. We denote the continuous functions on a set $S$ by $C^{0}(S)$; the continuous, non-negative functions on $S$ are denoted by $C_{+}^{0}(S)$. The non-negative functions with one continuous derivative on $S$ are denoted by $C_{+}^{1}(S)$. We denote the maximum norm in $C^{0}(S)$ by $\|\cdot\|$.

\section{A Boundary Value Problem for Some Ordinary Differential Equations}

We begin this section by studying the following boundary value problem involving only ordinary differential operators:

$$
\begin{aligned}
\frac{\partial f^{1}}{\partial x}+f^{1} f^{2} & =h^{1}, \quad f^{1}(0)=\varphi^{1}, \\
-\frac{\partial f^{2}}{\partial x}+f^{1} f^{2} & =h^{2}, \quad f^{2}(a)=\varphi^{2},
\end{aligned}
$$

where the functions $h$, as well as the boundary data $\varphi$ are given.

We prove

Lemma 2.1. Let $\varphi^{1}, \varphi^{2} \in \mathbb{R}_{+}^{1},\left(h^{1}, h^{2}\right) \in\left\{C_{+}^{0}[0, a]\right\}^{2}:=C_{+}^{0}[0, a] \times C_{+}^{0}[0, a]$. Then, the problem (2.1)-(2.2) has a unique solution $f=\left(f^{1}, f^{2}\right) \in\left\{C_{+}^{1}[0, a]\right\}^{2}$.

Proof. To solve (2.1)-(2.2), we use the method of Kaniel and Shinbrot [17], which is well suited to problems of this type. We begin by defining four sequences, $\left\{l_{n}^{1}\right\},\left\{u_{n}^{1}\right\}$, $\left\{l_{n}^{2}\right\}$, and $\left\{u_{n}^{2}\right\}$, as follows. Take $l_{0}^{1}=0=l_{0}^{2}$, and let $u_{0}^{1}$ and $u_{0}^{2}$ be the solutions of

$$
\begin{array}{cc}
\frac{\partial}{\partial x} u_{0}^{1}=h^{1}, & u^{1}(0)=\varphi^{1}, \\
\frac{\partial}{\partial x} u_{0}^{2}=-h^{2}, & u^{2}(a)=\varphi^{2} .
\end{array}
$$

\footnotetext{
${ }^{1}$ Although the corresponding one-dimensional Cauchy problem has been solved [3]
} 
Next, with $l_{n-1}^{1}, u_{n-1}^{1}, l_{n-1}^{2}$, and $u_{n-1}^{2}$ given, we define $l_{n}^{1}, u_{n}^{1}, l_{n}^{2}$, and $u_{n}^{2}$ as the solutions of the initial value problems

$$
\begin{aligned}
& \frac{\partial}{\partial x} u_{n}^{1}+l_{n-1}^{2} u_{n}^{1}=h^{1} \\
& \frac{\partial}{\partial x} l_{n}^{1}+u_{n-1}^{2} l_{n}^{1}=h^{1} \\
& l_{n}^{1}(0)=\varphi^{1}=u_{n}^{1}(0)
\end{aligned}
$$

and

$$
\begin{aligned}
& \frac{\partial}{\partial x} u_{n}^{2}-l_{n-1}^{1} u_{n}^{2}=-h^{2}, \\
& \frac{\partial}{\partial x} l_{n}^{2}-u_{n-1}^{1} l_{n}^{2}=-h^{2}, \\
& l_{n}^{2}(a)=\varphi^{2}=u_{n}^{2}(a) .
\end{aligned}
$$

A straightforward induction shows that

$$
0 \leqq l_{n}^{i} \leqq l_{n+1}^{i} \leqq u_{n+1}^{i} \leqq u_{n}^{i}, \quad i=1,2, n=0,1,2, \ldots .
$$

Thus, the sequences $\left\{l_{n}^{1}\right\}$ and $\left\{u_{n}^{1}\right\}$ are monotone and bounded, as are the sequences $\left\{l_{n}^{2}\right\}$ and $\left\{u_{n}^{2}\right\}$. All four are therefore convergent for each $x \in[0, a]$. Let $\left\{l_{n}^{i}\right\}$ converge to $l^{i},\left\{u_{n}^{i}\right\}$ to $u^{i}, i=1,2$. Integrating (2.3) and (2.4), we can send $n$ to infinity in the result to find that

$$
\begin{aligned}
& u^{1}(x)+\int_{0}^{x} l^{2}(\xi) u^{1}(\xi) d \xi=\varphi^{1}+\int_{0}^{x} h^{1}(\xi) d \xi, \\
& l^{1}(x)+\int_{0}^{x} u^{2}(\xi) l^{1}(\xi) d \xi=\varphi^{1}+\int_{0}^{x} h^{1}(\xi) d \xi, \\
& u^{2}(x)+\int_{x}^{a} l^{1}(\xi) u^{2}(\xi) d \xi=\varphi^{2}+\int_{x}^{a} h^{2}(\xi) d \xi, \\
& l^{2}(x)+\int_{x}^{a} u^{1}(\xi) l^{2}(\xi) d \xi=\varphi^{2}+\int_{x}^{a} h^{2}(\xi) d \xi .
\end{aligned}
$$

The functions $l^{1}, l^{2}, u^{1}, u^{2}$ are bounded, and these formulas show that they are absolutely continuous in $x$, and they satisfy

$$
\left.\begin{array}{c}
\frac{\partial}{\partial x} u^{1}+l^{2} u^{1}=h^{1}, \\
\frac{\partial}{\partial x} l^{1}+u^{2} l^{1}=h^{1}, \\
l^{1}(0)=\varphi^{1}=u^{1}(0), \\
\frac{\partial}{\partial x} u^{2}-l^{1} u^{2}=-h^{2}, \\
\frac{\partial}{\partial x} l^{2}-u^{1} l^{2}=-h^{2}, \\
l^{2}(a)=\varphi^{2}=u^{2}(a) .
\end{array}\right\}
$$


Thus, subtracting (2.5b) from (2.5a), and (2.6b) from (2.6a), we find

$$
\frac{\partial}{\partial x}\left(u^{1}-l^{1}\right)+l^{2}\left(u^{1}-l^{1}\right)=l^{1}\left(u^{2}-l^{2}\right)
$$

and

$$
\frac{\partial}{\partial x}\left(u^{2}-l^{2}\right)+l^{2}\left(u^{1}-l^{1}\right)=l^{1}\left(u^{2}-l^{2}\right)
$$

Thus,

$$
\frac{\partial}{\partial x}\left(u^{1}-l^{1}\right)=\frac{\partial}{\partial x}\left(u^{2}-l^{2}\right) .
$$

Since $\left(u^{1}-l^{1}\right)(0)=0$, then,

$$
\left(u^{1}-l^{1}\right)(x)=\left(u^{2}-l^{2}\right)(x)-\left(u^{2}-l^{2}\right)(0) .
$$

Setting $x=a$ and using the fact that $\left(u^{2}-l^{2}\right)(a)=0$, we find

$$
\left(u^{1}-l^{1}\right)(a)=-\left(u^{2}-l^{2}\right)(0),
$$

which is impossible unless both sides are zero. Thus, we have

$$
\left(u^{1}-l^{1}\right)(x)=\left(u^{2}-l^{2}\right)(x)
$$

with both sides being zero when $x=0$ or $x=a$. Equation (2.7) now gives

$$
\frac{\partial}{\partial x}\left(u^{1}-l^{1}\right)+\left(l^{2}-l^{1}\right)\left(u^{1}-l^{1}\right)=0 .
$$

Since $u^{1}-l^{1}=0$ when $x=0$, this means that $u^{1}=l^{1}$. Similarly, we find $u^{2}=l^{2}$. Writing $f^{1}$ for the common value of $l^{1}$ and $u^{1}$, and $f^{2}$ for $l^{2}$ and $u^{2}$, we find from (2.5) that $\left(f^{1}, f^{2}\right)$ is a solution of (2.1) and (2.2). Since $\left(f^{1}, f^{2}\right)$ satisfy

$$
\begin{aligned}
& f^{1}(x)+\int_{0}^{x} f^{2}(\xi) f^{1}(\xi) d \xi=\varphi^{1}+\int_{0}^{x} h^{1}(\xi) d \xi, \\
& f^{2}(x)+\int_{x}^{a} f^{2}(\xi) f^{1}(\xi) d \xi=\varphi^{2}+\int_{x}^{a} h^{2}(\xi) d \xi,
\end{aligned}
$$

they are absolutely continuous, and these equations can be differentiated to produce (2.1) and (2.2). This shows that Eq. (2.1)-(2.2), integrated with respect to $x$, have non-negative solutions $f^{1}, f^{2} \in L^{\infty}(0, a)$. However, integration of (2.1)-(2.2) gives a representation of $f^{1}$ and $f^{2}$ as integrals; thus, $f^{1}$ and $f^{2}$ are absolutely continuous. This fact used in (2.1)-(2.2) again shows that $\left(f^{1}, f^{2}\right) \in\left\{C_{+}^{1}[0, a]\right\}^{2}$. This completes the proof of existence.

As for uniqueness, notice that any solution of (2.1)-(2.2) in $\left\{C_{+}^{0}[0, a]\right\}^{2}$ satisfies $0=l_{0}^{i}(x) \leqq f^{i}(x) \leqq u_{0}^{i}(x), i=1,2$. An induction then shows that $l_{n}^{i}(x) \leqq f^{i}(x) \leqq u_{n}^{i}(x)$, $i=1,2, n=0,1, \ldots$. Since the sequences $\left\{l_{n}^{i}\right\}$ and $\left\{u_{n}^{i}\right\}$ converge to the solution constructed above, it follows that any solution is equal to the constructed solution. 
We now show that the solution of (2.1-2) delivered by Lemma 2.1 depends continuously on the data. For this, let $\left(f^{1}, f^{2}\right)$ satisfy $(2.1-2)$, and let $\left(F^{1}, F^{2}\right)$ be the solution of the same problem with different data, namely

$$
\begin{aligned}
\frac{\partial F^{1}}{\partial x}+F^{1} F^{2} & =H^{1}, & F^{1}(0) & =\Phi^{1}, \\
-\frac{\partial F^{2}}{\partial x}+F^{1} F^{2} & =H^{2}, & F^{2}(a) & =\Phi^{2} .
\end{aligned}
$$

We prove

Lemma 2.2. Let $\left(f^{1}, f^{2}\right)$ denote the solution of $(2.1)-(2.2),\left(F^{1}, F^{2}\right)$ the solution of (2.9)-(2.10). Take $\varepsilon>0$. Then, there exists a $\delta>0$ such that

$$
\left\|F^{1}-f^{1}\right\|+\left\|F^{2}-f^{2}\right\|<\varepsilon
$$

if

$$
\left\|H^{1}-h^{1}\right\|+\left\|H^{2}-h^{2}\right\|+\left|\Phi^{1}-\varphi^{1}\right|+\left|\Phi^{2}-\varphi^{2}\right|<\delta .
$$

$\varepsilon$ can be chosen to depend only on $\delta, a$, and the quantity

$$
\max \left[\left\|H^{1}\right\|,\left\|h^{1}\right\|,\left\|H^{2}\right\|,\left\|h^{2}\right\|,\left|\Phi^{1}\right|,\left|\Phi^{2}\right|,\left|\varphi^{1}\right|,\left|\varphi^{2}\right|\right) .
$$

Proof. Let

$$
c=\max \left[\left\|H^{1}\right\|,\left\|h^{1}\right\|,\left\|H^{2}\right\|,\left\|h^{2}\right\|,\left|\Phi^{1}\right|,\left|\Phi^{2}\right|,\left|\varphi^{1}\right|,\left|\varphi^{2}\right|\right) .
$$

We note first that the functions $f^{1}, f^{2}, F^{1}, F^{2}$ are all uniformly bounded, below by 0 and above by the corresponding function $u_{0}^{i}$ (see the proof of Lemma 2.1), which depends only on $c$ and $a$. Define $g^{1}=F^{1}-f^{1}, g^{2}=F^{2}-f^{2} \cdot g^{1}$ and $g^{2}$ satisfy

$$
\begin{array}{cc}
\frac{\partial g^{1}}{\partial x}+F^{2} g^{1}+f^{1} g^{2}=\eta^{1}, & g^{1}(0)=\psi^{1}, \\
-\frac{\partial g^{2}}{\partial x}+F^{2} g^{1}+f^{1} g^{2}=\eta^{2}, & g^{1}(0)=\psi^{2},
\end{array}
$$

where $\eta^{1}=H^{1}-h^{1}, \eta^{2}=H^{2}-h^{2}, \psi^{1}=\Phi^{1}-\varphi^{1}, \psi^{2}=\Phi^{2}-\varphi^{2}$. Subtracting (2.12) from (2.13) and integrating the result shows that there is a constant $c_{1}$ such that

$$
g^{1}(x)+g^{2}(x)=c_{1}+\int_{0}^{x}\left[\eta^{1}(\xi)-\eta^{2}(\xi)\right] d \xi:=c_{1}+\zeta(x),
$$

say. Solving (2.14) for $g^{2}$ and substituting into (2.12), we see that (2.12-13) is equivalent to the problem

$$
\begin{aligned}
\frac{\partial g^{1}}{\partial x}+\left(F^{2}-f^{1}\right) g^{1}+c_{1} f^{1} & =\eta^{1}-\zeta f^{1}, \\
: & =\eta .
\end{aligned}
$$

where $g^{1}(0)=\psi^{1}$ and $c_{1}$ must be chosen such that $g^{1}(a)=c_{1}-\psi^{2}+\eta(a)$. From (2.15), we obtain 


$$
g^{1}(a)=\psi^{1} e^{f^{\delta}\left(f^{1}-F^{2}\right)}+\int_{0}^{a}\left[\eta(x)-c_{1} f^{1}(x)\right] e^{\int_{x}^{a}\left(f^{1}-F^{2}\right)} d x
$$

The condition $g^{1}(a)=c_{1}-\psi^{2}+\eta(a)$ results in the equation

$$
\begin{aligned}
c_{1} & {\left[1+\int_{0}^{a} f^{1}(x) e^{\int_{x}^{a}\left(f^{1}-F^{2}\right)} d x\right] } \\
& =\psi^{2}-\zeta(a)+\int_{0}^{a} \eta(x) e^{\int_{x}^{a}\left(f^{1}-F^{2}\right)} d x+\psi^{1} e^{\int^{a}\left(f^{1}-F^{2}\right)} .
\end{aligned}
$$

Since $f^{1}(x) \geqq 0$, we read off immediately that

$$
\left|c_{1}\right| \leqq\left|\psi^{2}\right|+|\zeta(a)|+\int_{0}^{a}|\eta(x)| e^{\int_{x}^{a}\left(f^{1}-F^{2}\right)} d x+\left|\psi^{1}\right| e^{\int_{j}^{a}\left(f^{1}-F^{2}\right)} \leqq c_{2} \delta
$$

if (2.11) is satisfied. Here, $c_{2}$ is a constant depending only on $c$ and $a$. From (2.14), it follows that

$$
\left|\frac{\partial g^{1}}{\partial x}+\left(F^{2}-f^{1}\right) g^{1}\right| \leqq c_{3} \delta,
$$

where $c_{3}$ is another constant depending only on $c$ and $a$. Since $g^{1}$ satisfies

$$
\left|g^{1}(0)\right|=\left|\psi^{1}\right| \leqq \delta,
$$

the lemma follows.

Definition 2.3. For $\varrho>0$, let $B_{\varrho}^{0}$ denote the ball of radius $\varrho$ in $C^{0}(R),{ }_{+} B_{\varrho}^{0}$ the set of all non-negative elements of $B_{\varrho}^{0}$ :

$$
{ }_{+} B_{\varrho}^{0}=\left\{h \in C_{+}^{0}(R):\|h\| \leqq \varrho\right\} .
$$

Let $C^{01}(R)$ be the set of all functions continuous in $x$ and differentiable in $y$, normed by

$$
\|h\|_{01}=\|h\|+\|\partial h / \partial y\|
$$

$\|\cdot\|$ denoting the norm in $C^{0}(R)$, as before. Similarly, we define $C^{10}(R)$ as the set of functions differentiable in $x$ and continuous in $y$, with the norm

$$
\|h\|_{10}=\|h\|+\|\partial h / \partial x\| .
$$

We denote the balls of radius $\varrho$ in $C^{01}(R)$ and $C^{10}(R)$ by $B_{\varrho}^{01}$ and $B_{\varrho}^{10}$, respectively. ${ }_{+} B_{\varrho}^{01}$ and $+B_{\varrho}^{10}$ denote the non-negative functions in $B_{\varrho}^{01}$ and $B_{\varrho}^{10}$.

In Eqs. (2.1) and (2.2), we now allow the functions $h$ as well as the boundary data $\varphi$ to depend on $y$, and we prove

Lemma 2.4. Let $\left(\varphi^{1}, \varphi^{2}\right) \in\left\{C_{+}^{0}[0, b]\right\}^{2},\left(h^{1}, h^{2}\right) \in\left[C_{+}^{0}(R)\right]^{2}$. Then, Eqs. (2.1-2) have a unique solution $\left(f^{1}, f^{2}\right) \in\left[C_{+}^{0}(R)\right]^{2}$. With the boundary data $\left(\varphi^{1}, \varphi^{2}\right)$ fixed, let $S_{\varrho}$ denote the set of all solutions of (2.1-2) with $\left(h^{1}, h^{2}\right) \in{ }_{+} B_{\varrho}^{01}$. Then, the closure of $S_{\varrho}$ is a compact subset of $\left[C_{+}^{0}(R)\right]^{2}$. 
Proof. Applying Lemma 2.1 with $y \in[0, b]$ fixed, we find that $\left(f^{1}, f^{2}\right)$ exists for each $y$, and that $\left(f^{1}(\cdot, y), f^{2}(\cdot, y)\right) \in\left\{C_{+}^{1}[0, a]\right\}^{2}$. The continuity of the pair $\left(f^{1}, f^{2}\right)$ follows from Lemma 2.2 and the assumed continuity of the data.

For the compactness, we show that the set $S_{\rho}$ is equicontinuous. By Lemma 2.1, $f^{1}$ and $f^{2}$ are bounded. Equations (2.1) and (2.2) give, then, that the derivatives $\partial f^{1} / \partial x$ and $\partial f^{2} / \partial x$ are bounded. Lemma 2.2 shows that the moduli of continuity of $f^{1}$ and $f^{2}$ in the $y$-direction depend on the moduli of continuity of $\varphi^{1}, \varphi^{2}, h^{1}$, and $h^{2}$ in the $y$-direction. $\varphi^{1}$ and $\varphi^{2}$ are fixed, by hypothesis, while the moduli of continuity of $h^{1}$ and $h^{2}$ are controlled by their membership in $B_{0}^{01}$. Thus, the functions $f^{1}$ and $f^{2}$ vary over equicontinuous subsets of $C_{+}^{0}(R)$ as $h^{1}$ and $h^{2}$ vary over $+B_{\varrho}^{01}$. This completes the proof of the lemma.

One has merely to make the lexicographic change of replacing $x$ by $y$ and the indices 1 and 2 by 3 and 4 to prove

Lemma 2.5. Let $\left(\varphi^{3}, \varphi^{4}\right) \in\left\{C_{+}^{0}[0, a]\right\}^{2},\left(h^{3}, h^{4}\right) \in\left[C_{+}^{0}(R)\right]^{2}$. Then, the equations

$$
\begin{gathered}
\frac{\partial f^{3}}{\partial y}+f^{3} f^{4}=h^{3}, \quad f^{3}(0)=\varphi^{3}, \\
-\frac{\partial f^{4}}{\partial y}+f^{3} f^{4}=h^{4}, \quad f^{4}(a)=\varphi^{4},
\end{gathered}
$$

have a unique solution $\left(f^{3}, f^{4}\right) \in\left[C_{+}^{0}(R)\right]^{2}$. With the boundary data $\left(\varphi^{3}, \varphi^{4}\right)$ fixed, let $S_{\varrho}^{\prime}$ denote the set of all solutions of $(2.16-17)$ with $\left(h^{3}, h^{4}\right) \in+B_{\varrho}^{10}$. Then, the closure of $S_{\varrho}^{\prime}$ is a compact subset of $\left[C_{+}^{0}(R)\right]^{2}$.

\section{The Operator $T$ and Some of its Properties}

We now proceed to consider (1.1)-(1.4). For this, we assume once and for all that $\left(\varphi^{1}, \varphi^{2}, \varphi^{3}, \varphi^{4}\right)$ is fixed, satisfying the hypotheses of Lemmas 2.4 and 2.5 . We establish a mapping from the cone $\left[C_{+}^{0}(R)\right]^{4}$ into itself, as follows. Let $\left(g^{1}, g^{2}, g^{3}, g^{4}\right) \in\left[C_{+}^{0}(R)\right]^{4}$. Solve the equations

$$
\begin{array}{rr}
\frac{\partial f^{1}}{\partial x}+f^{1} f^{2}=g^{3} g^{4}, & f^{1}(0, y)=\varphi^{1}(y), \\
-\frac{\partial f^{2}}{\partial x}+f^{2} f^{2}=g^{3} g^{4}, & f^{2}(a, y)=\varphi^{2}(y), \\
\frac{\partial f^{3}}{\partial y}+f^{3} f^{4}=g^{1} g^{2}, & f^{3}(x, 0)=\varphi^{3}(x), \\
-\frac{\partial f^{4}}{\partial y}+f^{3} f^{4}=g^{1} g^{2}, & f^{4}(x, b)=\varphi^{4}(x) .
\end{array}
$$

We prove

Lemma 3.1. Let $\left(g^{1}, g^{2}, g^{3}, g^{4}\right) \in\left[C_{+}^{0}(R)\right]^{4}$. Then, Eqs. (3.1)-(3.4) have a solution $f=\left(f^{1}, f^{2}, f^{3}, f^{4}\right) \in\left[C_{+}^{0}(R)\right]^{4}$. 
Proof. Equations (3.1)-(3.2) are subject to Lemma 2.4, Eqs. (3.3)-(3.4) to Lemma 2.5. The result follows immediately.

Definition 3.2. As the proof of Lemma 3.1 shows, the pair of Eqs. (3.1)-(3.2) can be solved independently of the pair (3.3)-(3.4). Thus, solving (3.1)-(3.2) defines a mapping of the pair $\left(g^{3}, g^{4}\right)$ into the solution pair $\left(f^{1}, f^{2}\right)$. We write $\left(f^{1}, f^{2}\right)$ $=T_{x}\left(g^{3}, g^{4}\right)$; the subscript $x$ is used to indicate that $T_{x}$ is smoothing with respect to the variable $x$ (since the image of $\left[C_{+}^{0}(R)\right]^{2}$ under $T_{x}$ is contained in $\left[C_{+}^{10}(R)\right]^{2}$ ). In a similar way, and for similar reasons, we write $\left(f^{3}, f^{4}\right)=T_{y}\left(g^{1}, g^{2}\right)$ if $\left(f^{3}, f^{4}\right)$ is the solution of (3.3)-(3.4). Finally, we write $T\left(g^{1}, g^{2}, g^{3}, g^{4}\right)=\left(f^{1}, f^{2}, f^{3}, f^{4}\right)$ for the full solution of (3.1)-(3.4). Any solution of (1.1)-(1.4) is a fixed point of $T$.

We begin our study of the operator $T$ with

Lemma 3.3. The operator $T^{2}$ is compact.

Proof. We have, in the obvious notation,

$$
T g=\left(T_{x}\left(g^{3}, g^{4}\right), T_{y}\left(g^{1}, g^{2}\right)\right) \text {. }
$$

Accordingly,

$$
T^{2} g=\left(T_{x} T_{y}\left(g^{1}, g^{2}\right), T_{y} T_{x}\left(g^{3}, g^{4}\right)\right) .
$$

We show that $T_{x} T_{y}$ is compact on $\left[C_{+}^{0}(R)\right]^{2}$. $T_{y}$ maps $\left[C_{+}^{0}(R)\right]^{2}$ into $\left[C_{+}^{01}(R)\right]^{2}$. Accordingly, if $\left(g^{1}, g^{2}\right)$ varies over a bounded subset of $\left[C_{+}^{0}(R)\right]^{2}$, all the images $T_{y}\left(g^{1}, g^{2}\right)$ lie in a ball $\left[{ }_{+} B_{\varrho}^{01}\right]^{2}$, for some $\varrho>0$. Lemma 2.4 therefore shows that $T_{x} T_{y}$ is compact. A similar argument, using Lemma 2.5, shows that $T_{y} T_{x}$ is compact also, and the result follows from this.

Next, we prove

Lemma 3.3. The operator $T^{2}$ has a fixed point in $\left[C_{+}^{0}(R)\right]^{4}$.

Proof. According to the theorem of Schaefer ${ }^{2}[18,19]$, we have to show that any solution of $f=\lambda T^{2} f$ with $0<\lambda<1$ is bounded. Suppose $f=\lambda T^{2} f$. Then, (3.5) gives

$$
\left(f^{1}, f^{2}\right)=\lambda T_{x} T_{y}\left(f^{1}, f^{2}\right)
$$

and

$$
\left(f^{3}, f^{4}\right)=\lambda T_{y} T_{x}\left(f^{3}, f^{4}\right) .
$$

We show first that $f^{1}$ is bounded. Let

$$
\left(g^{3}, g^{4}\right)=T_{y}\left(f^{1}, f^{2}\right) .
$$

Then, according to (3.6),

$$
\left(f^{1}, f^{2}\right)=\lambda T_{x}\left(g^{3}, g^{4}\right)
$$

\footnotetext{
${ }^{2}$ Schaefer's theorem refers to a mapping of an entire Banach space into itself. However, the proof depends on a retraction of the operator under consideration. As a consequence, it is easy to extend the proof to apply to a mapping on a cone, like $\left[C_{+}^{0}(R)\right]^{2}$
} 
The definitions of $T_{x}$ and $T_{y}$ give

$$
\begin{aligned}
& \frac{\partial f^{1}}{\partial x}=\lambda g^{3} g^{4}-\frac{1}{\lambda} f^{1} f^{2}, f^{1}(0, y)=\lambda \varphi^{1}(y), \\
& \frac{\partial f^{2}}{\partial x}=\frac{1}{\lambda} f^{1} f^{2}-\lambda g^{3} g^{4}, f^{2}(a, y)=\lambda \varphi^{2}(y), \\
& \frac{\partial g^{3}}{\partial y}=f^{1} f^{2}-g^{3} g^{4}, \quad g^{3}(x, 0)=\varphi^{3}(x), \\
& \frac{\partial g^{4}}{\partial y}=g^{3} g^{4}-f^{1} f^{2}, \quad g^{4}(x, b)=\varphi^{4}(x)
\end{aligned}
$$

Therefore,

$$
\begin{aligned}
\frac{\partial}{\partial x}\left(f^{1}-f^{2}\right)+\frac{\partial}{\partial y}\left(g^{3}-g^{4}\right) & =2(\lambda-1) g^{3} g^{4}+2\left(1-\frac{1}{\lambda}\right) f^{1} f^{2} \\
& \leqq 0
\end{aligned}
$$

since $0<\lambda<1$, while the functions $f$ and $g$ are all non-negative. Integrating (3.11) over $R$ and using the divergence theorem, we find

$$
\int_{x=a}\left(f^{1}-f^{2}\right) d y-\int_{x=0}\left(f^{1}-f^{2}\right) d y+\int_{y=b}\left(g^{3}-g^{4}\right) d x-\int_{y=0}\left(g^{3}-g^{4}\right) d x \leqq 0 .
$$

Using the boundary conditions on $f^{1}, f^{2}, g^{3}$, and $g^{4}$, we find

$$
\begin{aligned}
& \int_{x=a} f^{1} d y+\int_{x=0} f^{2} d y+\int_{y=b} g^{3} d x+\int_{y=0} g^{4} d x \\
& \quad \leqq \int_{x=0} \lambda \varphi^{1}(y) d y+\int_{x=a} \lambda \varphi^{2}(y) d y+\int_{y=0} \varphi^{3} d x+\int_{y=b} d x \\
& \quad \leqq \int_{\partial R} \varphi d s,
\end{aligned}
$$

again using the fact that $0<\lambda<1$. Here, $s$ denotes arclength on $\partial R$, and $\varphi: \partial R \rightarrow \mathbb{R}_{+}^{1}$ is $\varphi^{1}$ on the left side of $R, \varphi^{2}$ on the right, $\varphi^{3}$ on the bottom and $\varphi^{4}$ on the top. Equation (3.12) gives $L^{1}$ bounds on the functions $f^{1}, f^{2}, g^{3}, g^{4}$ on the part of the boundary of $R$ opposite to that of the data.

The inequality (3.12) follows from mass conservation. We now return to (3.7) $-(3.10)$ and use momentum conservation in the form

$$
\begin{aligned}
& \frac{\partial}{\partial x}\left(f^{1}+f^{2}\right)=0 \\
& \frac{\partial}{\partial y}\left(g^{3}+g^{4}\right)=0
\end{aligned}
$$

Equation (3.13) shows that $f^{1}+f^{2}$ is a function of $y$ alone and $g^{3}+g^{4}$ is a function of $x$ alone. Now, choose $\bar{x} \in[0, a]$ arbitrarily and choose an interval $I \subset[0, b]$. Integrating $(3.13 \mathrm{a})$ over $[0, \bar{x}] \times I$, we find

$$
\begin{aligned}
\int_{I}\left(f^{1}+f^{2}\right)(\bar{x}, y) d y & =\int_{I}\left(f^{1}+f^{2}\right)(0, y) d y . \\
& =\int_{I}\left[\varphi^{1}(y)+f^{2}(0, y)\right] d y \\
& \leqq 2 \int_{\partial R} \varphi d s,
\end{aligned}
$$


by (3.12). Let $\Gamma$ be any positive constant. Since the left side of (3.14) is independent of $\bar{x}$, it follows that we can partition the interval $[0, b]$ by $n+1$ points $0=y_{0}<y_{1}<\ldots y_{n}=b$, also independent of $\bar{x}$, in such a way that

$$
\int_{y_{l}}^{y_{2}+1}\left(f^{1}+f^{2}\right)(\bar{x}, y) d y<\frac{1}{2 \Gamma},
$$

and $n \leqq 2 \Gamma \int_{\partial R} \varphi d s+1$.

The same argument shows that the interval $[0, a]$ can be partitioned by $m+1$ points $0=x_{0}<x_{1}<\ldots<x_{m}=a$ such that for any $\bar{y} \in[0, b]$,

$$
\int_{x_{i}}^{x_{i}+1}\left(g^{3}+g^{4}\right)(x, \bar{y}) d x<\frac{1}{2 \Gamma},
$$

where $m \leqq 2 \Gamma \int_{\partial R} \varphi d s+1$

We now use these estimates to obtain pointwise bounds on $f^{1}$. From (3.7), we have

$$
f^{1}(x, y) \leqq \lambda \varphi^{1}(y)+\lambda \int_{0}^{x} g^{3} g^{4}(\sigma, y) d \sigma
$$

Next, we use (3.9) to estimate $g^{3}$, and insert the result in this last inequality. We find

$$
\begin{aligned}
f^{1}(x, y) \leqq & \lambda \varphi^{1}(y)+\lambda \int_{0}^{x} g^{4}(\sigma, y) \varphi^{3}(\sigma) d \sigma \\
& +\lambda \int_{0}^{x} \int_{0}^{y} g^{4}(\sigma, y)\left(f^{1} f^{2}\right)(\sigma, \tau) d \tau d \sigma .
\end{aligned}
$$

Let

$$
K(x)=\max _{\substack{0 \leqq \xi \leqq x \\ 0 \leqq y \leqq b}} f^{1}(\xi, y) .
$$

We show that $K(x)$ is bounded by a constant depending only on the data. In the rest of the proof, we reserve the letter $c$ to denote such a constant; $c$ may have different values in different formulas.

As $\lambda<1$, we have $\lambda \varphi^{1}(y), \lambda \varphi^{3} \leqq c$. By (3.17), then,

$$
\lambda \int_{0}^{x} g^{4}(\sigma, y) \varphi^{3}(\sigma) d \sigma \leqq c .
$$

Also, (3.14) shows that

$$
\int_{0}^{y} f^{2}(\sigma, \tau) d \tau \leqq c \quad \text { for all } \sigma .
$$

We apply the definition of $K$ and these estimates to the right side of (3.18) to find

$$
f^{1}(x, y) \leqq c\left[1+K(x) \int_{0}^{x} g^{4}(\sigma, y) d \sigma\right] .
$$

Now, take $\Gamma$ equal to this last constant $c$, and choose the partition in (3.17) corresponding to this value of $\Gamma$. We see then that

$$
\int_{0}^{x} g^{4}(\sigma, y) d \sigma \leqq \frac{1}{2 \Gamma} \text { if } \quad 0 \leqq x \leqq x_{1}
$$


and from (3.19), we conclude that

$$
K(x) \leqq \Gamma+\frac{1}{2} K(x)
$$

that is,

$$
K(x) \leqq 2 \Gamma, \quad 0 \leqq x \leqq x_{1} .
$$

This is a bound for $f^{1}$ on the rectangle $\left[0, x_{1}\right] \times[0, b]$. Repeating the argument in the rectangle $\left[x_{1}, x_{2}\right] \times[0, b]$, we find

$$
K(x) \leqq 4 \Gamma, \text { for } x_{1} \leqq x \leqq x_{2},
$$

and, inductively, we prove

$$
K(x) \leqq 2^{m} \Gamma \text { for } 0 \leqq x \leqq a .
$$

Since $m$ depends only on $\Gamma$ and $\Gamma$ depends only on the data, the estimate on $f^{1}$ is complete. $f^{2}, g^{3}$, and $g^{4}$ are estimated in a similar way. Schaefer's theorem thus implies the result.

\section{The Main Result}

A solution of the problem (1.1)-(1.4) is a fixed point of $T$. We show in this section that such a fixed point exists.

Theorem 4.1. Let $\left(\varphi^{1}, \varphi^{2}\right) \in\left\{C_{+}^{0}[0, b]\right\}^{2},\left(\varphi^{3}, \varphi^{4}\right) \in\left\{C_{+}^{0}[0, a]\right\}^{2}$. Then, the problem (1.1)-(1.4) has a solution $f=\left(f^{1}, f^{2}, f^{3}, f^{4}\right) \in\left[C_{+}^{0}(R)\right]^{4}$.

Proof. Let $h=\left(h^{1}, h^{2}, h^{3}, h^{4}\right)$ be the fixed point of $T^{2}$ guaranteed by Lemma 3.3. Then, we have

$$
\left(h^{1}, h^{2}\right)=T_{x} T_{y}\left(h^{1}, h^{2}\right)
$$

Set

$$
\left(g^{3}, g^{4}\right)=T_{y}\left(h^{1}, h^{2}\right)
$$

Then,

$$
\left(h^{1}, h^{2}\right)=T_{x}\left(g^{3}, g^{4}\right)
$$

Since $T\left(f^{1}, f^{2}, f^{3}, f^{4}\right)=\left(T_{x}\left(f^{3}, f^{4}\right), T_{y}\left(f^{1}, f^{2}\right)\right)$, as we saw in Lemma 3.1 , the function $f=\left(h^{1}, h^{2}, g^{3}, g^{4}\right)$ is a fixed point of $T$ and a solution of the problem.

\section{References}

1. Arthur, M.D., Cercignani, C.: Nonexistence of a steady rarefied supersonic flow in a halfspace. Z. Angew. Math. Phys. 31, 634 (1980)

2. Bardos, C., Caflisch, R.E., Nicolaenko, B.: The Milne and Kramers problems for the Boltzmann equation of a hard sphere gas. Commun. Pure Appl. Math. 39, 322 (1986)

3. Beale, J.T.: Large-time behavior of the Broadwell model of a discrete velocity gas. Commun. Math. Phys. 102, 217-235 (1985) 
4. Beals, R.: An abstract treatment of some forward-backward problems of transport and scattering. J. Funct. Anal. 34, 1 (1979)

5. Cercignani, C.: Mathematical methods in kinetic theory. New York: Plenum Press 1969

6. Cercignani, C.: Theory and application of the Boltzmann equation. New York: Elsevier 1975

7. Cercignani, C.: Elementary solutions of the linearized gas dynamics Boltzmann equation and their application to the slip flow problem. Ann. Phys. (NY) 20, 219 (1962)

8. Cercignani, C.: Existence and uniqueness in the large for boundary value problems in kinetic theory. J. Math. Phys. 8, 1653-1656 (1967)

9. Cercignani, C.: On the general solution of the steady linearized Boltzmann equation. In: Rarefied gas dynamics. Becker, M., Fiebig, M. (eds.). Porz-Wahn: DFVLR Press 1974

10. Cercignani, C.: Half-space problems in the kinetic theory of gases. In: Trends in applications of pure mathematics to mechanics. Kröner, E., Kirchgässer, K. (eds.), Lecture Notes in Physics, Vol. 249, p. 35. Berlin, Heidelberg, New York: Springer 1986

11. Cercignani, C., Illner, R., Shinbrot, M.: A boundary value problem for discrete velocity models. Duke Math. J. (to appear)

12. Cercignani, C., Illner, R., Pulvirenti, M., Shinbrot, M.: On nonlinear stationary half-space problems in discrete kinetic theory (to appear)

13. Gatignol, R.: Théorie Cinétique des gaz a répartition discréte des vitésses. Lecture Notes in Physics, Vol. 36. Berlin, Heidelberg, New York: Springer 1975

14. Grcenberg, W., van der Mee, C.: An abstract approach to evaporation models in rarefied gas dynamics. Z. Angew. Math. Phys. 35, 166 (1984)

15. Illner, R.: Global existence results for discrete velocity models of the Boltzmann equation in several dimensions. J. Méc. Théor. Appl. 1, 611-622 (1982)

16. Illner, R.: Examples of non-bounded solutions in discrete kinetic theory. J. Méc. Théor. Appl. 5, 561-571 (1986)

17. Kaniel, S., Shinbrot, M.: The Boltzmann equation. I. Uniqueness and local existence. Commun. Math. Phys. 58, 65-84 (1978)

18. Schaefer, H.: Über die Methode der a priori Schranken. Math. Ann. 129, 415-416 (1955)

19. Smart, D.R.: Fixed point theorems. New York: Cambridge University Press 1974

Communicated by J. L. Lebowitz

Received July 14, 1987 